\title{
A Taxonomic Study of Macro-and Micro Morphological Features of Fruits and Seeds Cultivars of the Species Belonging to the Genus Morus L. (Moraceae) Cultivated in the North of Iraq
}

\author{
Raad Hamad Mahmoud Al-Badrany ${ }^{1 *}$, Amer Mohsen Mahmoud Al-Mathidy ${ }^{2}$ \\ ${ }^{1,2}$ Biology Department, College of Education for Pure science, University of Mosul, Mosul, Iraq \\ E-mail: ${ }^{1 *}$ raadhamad42@gmail.com, ${ }^{2}$ dr.amer.1956@yahoo.com
}

(Received May 31, 2020; Accepted July 26, 2020; Available online December 01, 2020)

DOI: 10.33899/edusj.2020.127107.1074, (C) 2020, College of Education for Pure Science, University of Mosul.

This is an open access article under the CC BY 4.0 license (http://creativecommons.org/licenses/by/4.0/).

\begin{abstract}
:
The present research deals with the study of morphological characters of the fruits and seeds of the cultivars belonging to the species Morus alba L. Namely ('Beautiful Day', 'Big White', 'Rease', 'Greece', 'Pearl', 'Border Sweet', 'Pendula'); Morus latifolia Poir ('Kokuse Korean'); Morus rubra L. ('Amarah'); Morus nigra L. ('Shami'); Morus macroura Miq ('King White'); 'Dwarf' and Morus hybrid ('Tice', 'Wellington') Which cultirated in North of Iraq.

The study includes characters of the fruits (Shape, Color, Dimension, Size, Number of the fruitlet in the fruit) and the characters of the seeds (Shape, Color, Dimension, Number of the seeds in the fruit, and surface ornamentation).

The results showed some changes in morphological characteristics of the fruits and seeds examined by light microscope (L.M), in addition to the surface ornamentation of the seeds examined by scanning electron microscope (SEM). They also showed five types of the surface ornamentation (Reticulate, Muricate, Foreate undulate, Polygonal, and Ruminate) for cultivars species studied, showed that importance value in the separation between the cultivars of the species studied belonging to the genus Morus L.
\end{abstract}

Keyword: Morphological, Systematic, Fruits, Seeds, Genus Morus L.

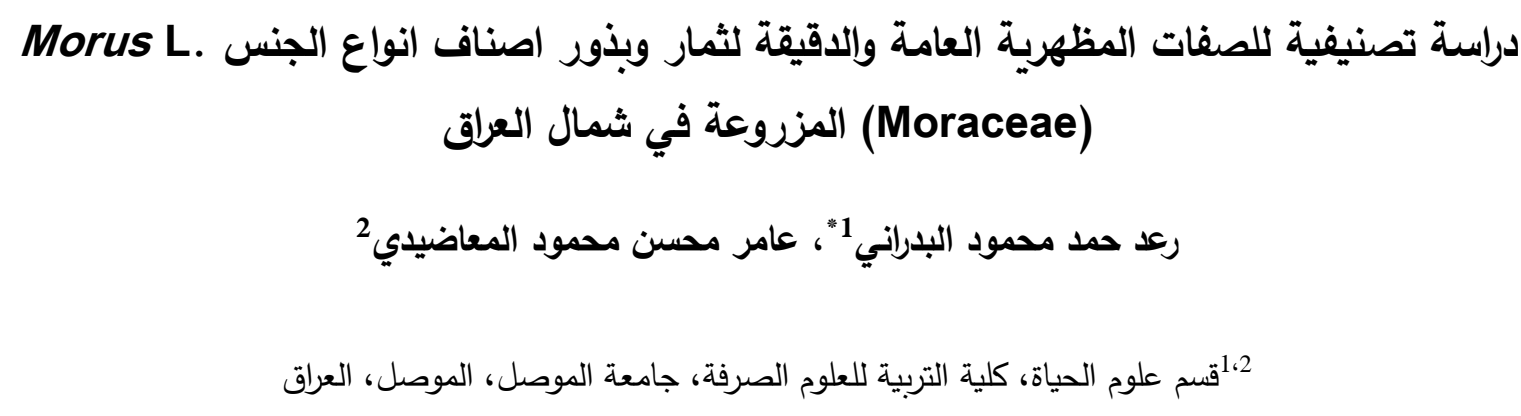

الخلاصة:

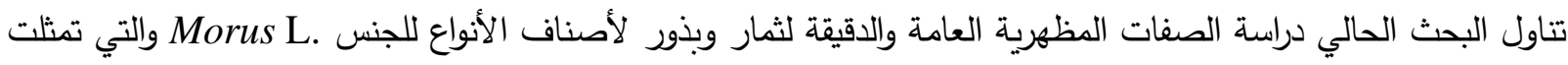

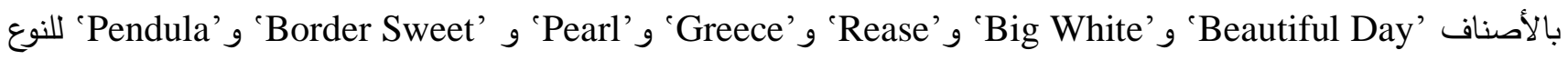

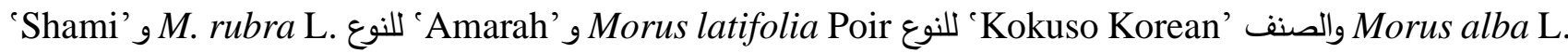




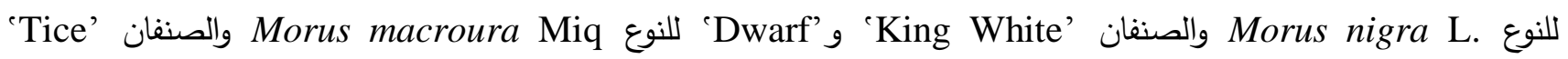
و'Morus hybrid للنوع 'Wellington' المزروعة في شمال العراق. وتضمنت الدراسة صفات الثمار من حيث شكلها ولونها وابعادها وحجمها وعدد الثميرات في الثمرة الواحدة وصفات البذور من

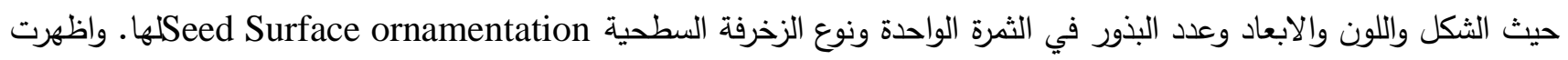

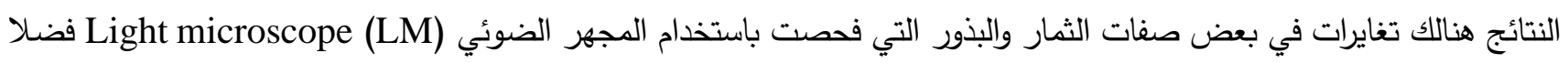
عن الكساء السطحي للبذور بينت الأصناف في زخرفتها السطحية Surface ornamentation وباستخدام المجهر الاككتروني الماسح Scanning Electron Microscope (SEM) Muricate ومتموج منقر Poreate undulate ومضلع Polygonal ومنسحب Ruminate في أنوع الأصناف قيد الدراسة والتي وهي لها أهمية تصنيفية في عزل الأصناف بين الأنواع المدروسة والعائدة الى جنس .Morus L.

Morus L. الكلمات المفتاحية: المظهرية، التصنيف، الثمار، البذور، جنس

المقدمة

ان جنس التوت .Morus L والذي ينتمي إلى العائلة التوتية Roraceae رتبة Rosales ينمو بشكل اشجار وشجيرات معرة،

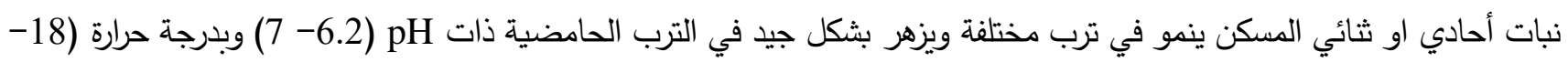
30 C (C)، ويعد معقد وراثياً وله قابلية كبيرة على التغاير والتكيف والانتشار على نطاق واسع في البيئات المختلفة [2،1]. وتعد الصفات المظهرية للنباتات القاعدة الاساس لوصف المجاميع النباتية وتثخيصها بالرغم من ظهور الدراسات الحديثة وتطور الوسائل العلمية التي فتحت افاقاً واسعة في الدراسات التصنيفية، احتفظت هذه الصفات بمنزله الصدارة وذلك لاككانية تمييزها

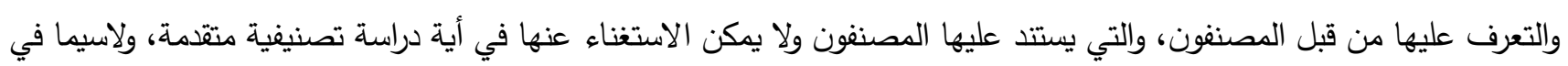

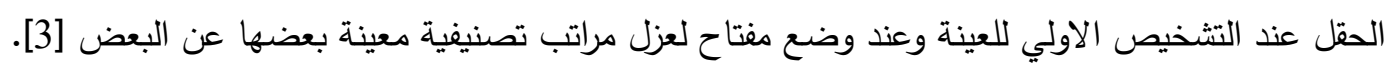
وبينّ [4] أن الأدلة التصنيفية لجميع أجزاء النبات خلال فترة نموه ومراحل تكثفه ذات أهمية تصنيفية وأن المؤشرات المظهرية لتوصيف الاصناف الجديدة لكل نوع تعتمد على مجموعة من الصفات التي لها القدرة في تمييز الاصناف بعضها عن البعض. أن [5] شخص 34 صنفاً من التوت النامي في تركيا بالاعتماد على الصفات المظهرية للثمار اذ لاحظ وجود اختلافات كبيرة

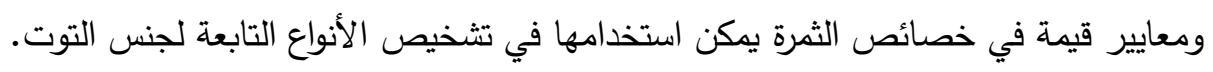
كما استخدم [6] الصفات المظهرية للثمار في تثخيص وتصنيف التراكيب الوراثية Genotypes للتوت الابيض

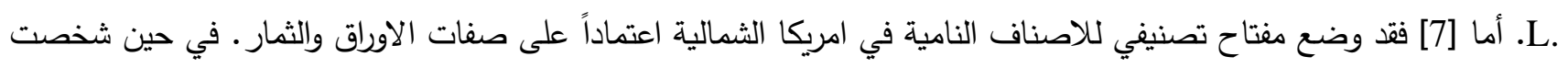
[8] احدى وثلاثون صنفاً من التوت باستخدام الصفات المظهرية للثمار الكمية والنوعية.

وان للزخرفة والعلامات السطحية للبذرة أهمية في تصنيف الاجناس والانواع والاصناف التابعة لها خصوصا بعد استخدام

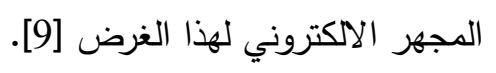

وتمكن [10] من تشخيص بذور 5 اصناف من التوت الابيض .M. alba L باستخدام الصفات المظهرية للبذرة مثل طول وعرض وسمك البذرة بالاضافة إلى صفات جنين البذرة وأثار بأن لها فائدة في الحفاظ على بنك المورثات لألى للاكثار الجنسي. 
وأورد[11] إلى أن الالتباس الذي يحدث في يومنا هذا في تقسيمات التوت يؤكد ضرورة انشاء تتوصيف له للمساعدة في

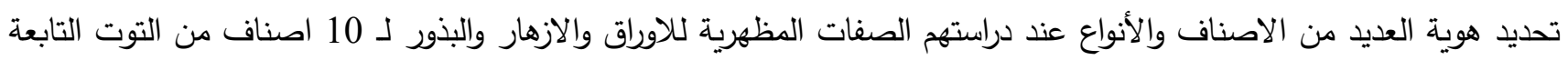
للنوعين M. abla و M. Latifolia المستزرعة في ايطاليا باستخدام المجهر الالكتروني الماسح (SEM). في الآونة الاخيرة ازداد الاهتمام الكبير بزراعة اشجار التوت واستهلاك فاكهته بشكل سريع، لما لها من طعم جيد وقيمة غذائية

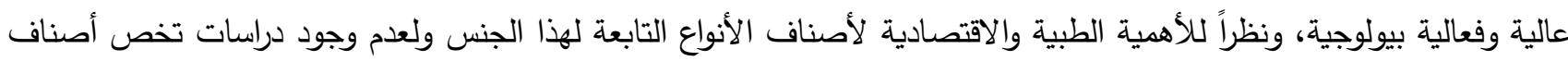

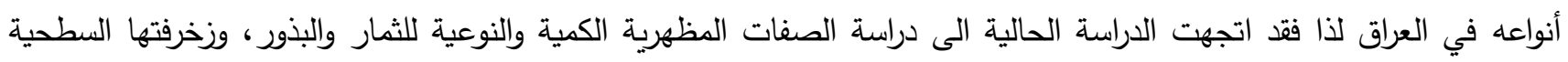

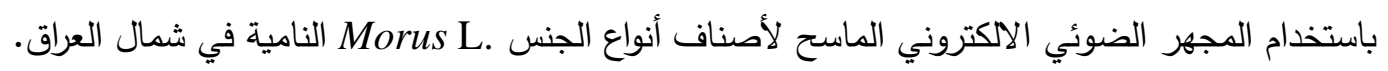

\section{Materials and Methods المواد وطرائق العمل}

إعتمدت الدراسة على العينات الطرية لأصناف أنواع الجنس .Morus L المدروسة والتي جمعت من عدّة مناطق من شمال العراق خلال المسح الميداني في محافظتي نينوى ودهوك التي شملت محطة بستتة نينوى ودهوك، والمشاتل والحقول التابعة لها. والمشخصة من قبل وزارة الزراعة والمستزرعة في تلك المناطق وخلال فترة النمو لعامي 2018-2019. اذ تم اختيار خمس أشجار توت من كل صنف متجانسة قدر الإمكان في قوة النمو والعمر • جمعت العينات وصورت بشكل

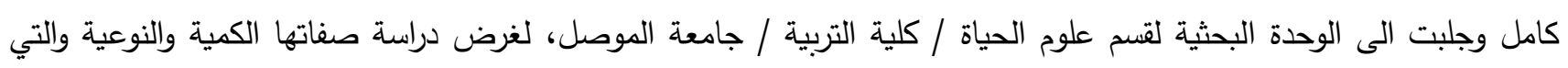
شملت:

1.

A . ابعاد الثمرة: تم قياس طول الثمرة وعرضها بواسطة القدمة الالكترونية (Vernier) بوحدة (cm).

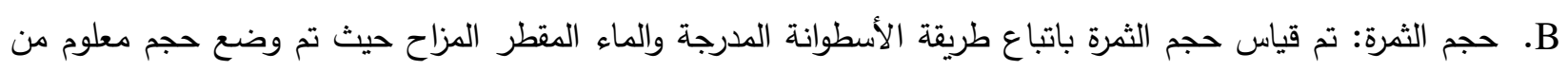
الماء المقطر في الأسطوانة المدرجة وغمرت الثمرة داخل الأسطوانة وتم قياس الحجم عن طريق إيجاد الفرق بين مستوى

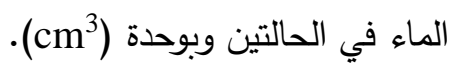
C . شكل الثمرة ولونها: تم تحديد شكل الثمرة ولونها باستخدام العين المجردة. D. عدد الثميرات/ثمرة: تم حساب عدد الثميرات يدويا وذلك بفصلها بملقط دقيق من الثمرة. 2. A. البعاد البذور: تمت دراسة طول البذرة وعرضها وسمكها باستخدام الورق البياني ومجهر تشريح ضوئي 15X نوع wild

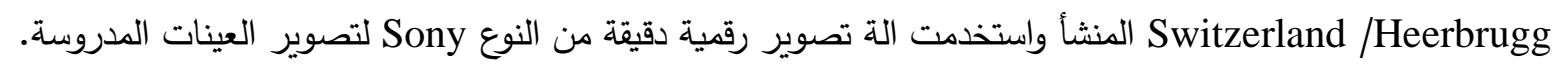
B. شكل البذور ولونها: تم تحديد شكل البذرة ولونها بعد تكبيرها بالمجهر التشريحي الضوئي 15X لصغر حجمها وصورت

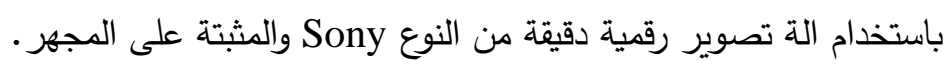
C عدد البذور بالثمرة: تم حساب عدد البذور /ثمرة يدويا.

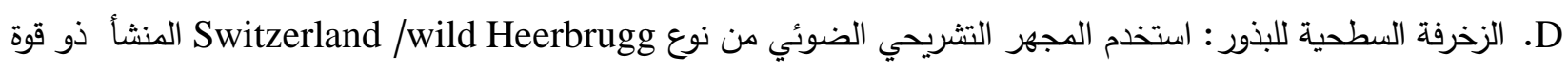

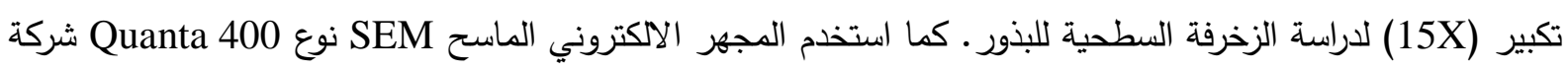
هولندية المنشأ اذ درست الزخرفة السطحية للبذور Sculpture seeds surface في جامعة السليمانية/ كلية العلوم/

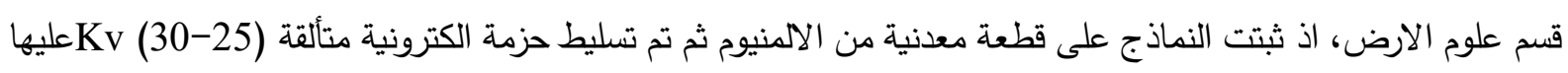
ثم تغيير مجال الفحص في المجهر الالكتروني للحصول على صور واضحة بشكل دقيق. 
تم اخذ القياسات لـ 25 ثمرة و25 بذرة من كل صنف ولكل صفة، وتم أخذ معدلات القياسات وإيجاد الانحراف المعياري لها، وتمت جدولة البيانات لمختلف الصفات المظهرية بعد تحويلها الى نتائج كمية ونوعية لغرض المقارنة بين أصناف الأنواع قيد الدراسة اعتمادا على أساس التثابه والاختلاف بالنسبة للصفات الكمية والنوعية المدروسة.

Results and Discussions النتائج والمناقثة

$$
\text { 1- الاجزاء الثمرية: }
$$

Fruiting stalk الحامل الثمري. A

'Shami' اظهرت الدراسة وجود تغايرات في الحامل الثمري في اصناف الانواع اذ كانت الثمار جالسة Sessile في الصنف

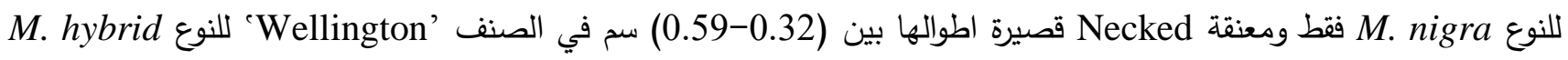
M. latifolia وجميع اصناف النوع Morder Sweet ومدا الصنف

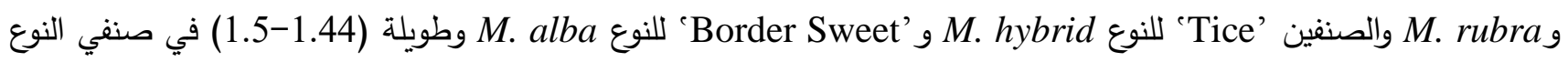
M. macroura

Fruits الثمار

بينت الدراسة أن ثمار اصناف الانواع من الثمار المتضاعفة Multiple fruits (المركبة Compound) والتي تعرف بـ Sorosis بعضها البعض وحتى ضمن النوع الواحد اذ أمكن تقسيم الثمار استناداً لشكلها الى خمس مجاميع، كما مبين في الجدول (1) وانئ والثكل : (1) المجموعة الأولى: شبه كروية - S- Semispherical - Elliptic وضليجية 'Shami' للنوع الصنف .M. nigra

المجموعة الثانية: بيضية Ovoid وضمت ثلاثة اصناف للنوع M. alba وهي 'Greece و'Pearl و و'Pendula'. المجموعة الثالثة: بيضية متطاولة Elongate ovoid وشملت الصنف 'Kokuso Korean’ للنوع M.latifolia والاصناف .M. hybrid للنوع 'Wellington' و M.alba للنوع 'BigWhite' المجموعة الرابعة: أهليجية Elliptic وتضم صنف 'Amarah' النوع M. rubra والصنف 'Tice للنوع Mybrid وبقية اصناف النوع M. alba قيد الدراسة.

المجموعة الخامسة: اسطوانية Clyindric وتمثلت بالصنفان 'Ding White' و' للنوع 'Dwacroura.

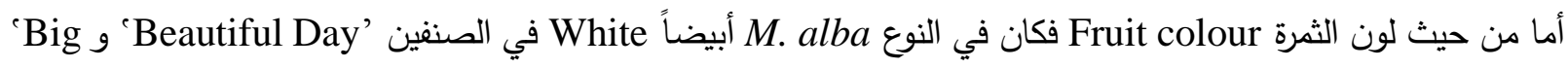

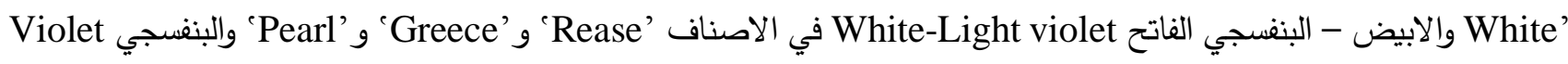

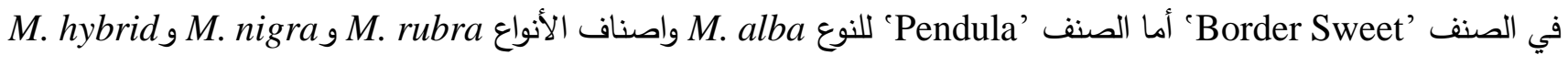

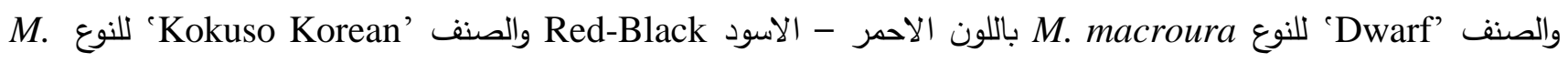
Greenish والصنف 'King White للنوع Macroura violet باللون البنفجي الداكن latifolia ·yellow 
بالاضافة الى ذلك اختلفت الاصناف تحت الدراسة في صفة عدد الثميرات Number fruitlet في الثمرة الواحدة اذ بلغ معدله في حده الأدنى (22.3) ثميرة/ ثمرة في صنف النوع Migra وفي حده الأعلى (127.5) ثميرة/ ثمرة في الصنف 'King White'

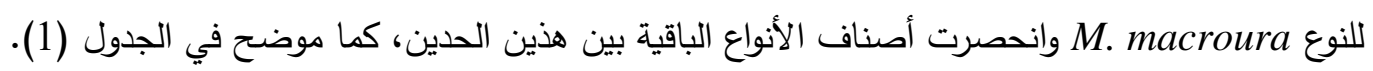
أما صفة طول الثمرة Length fruit فتعد من الصفات التثخيصية المهمة حيث أمكن عزل اصناف الأنواع قيد الدراسة الى ثلاث مجاميع وهي:

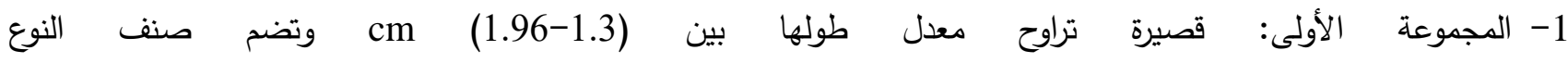

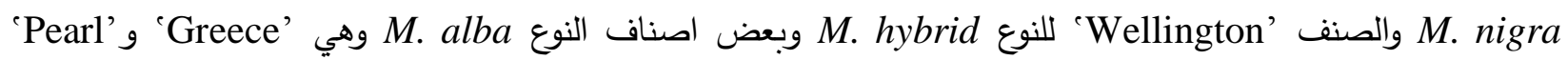
.Pendula'g 'Border Sweet'

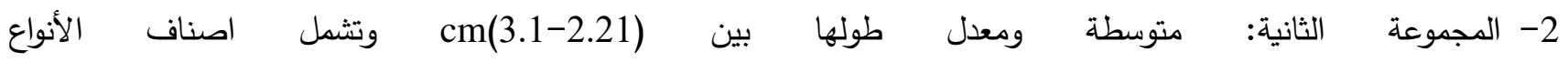

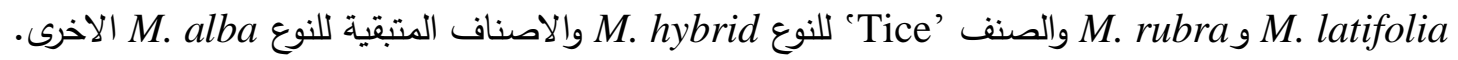

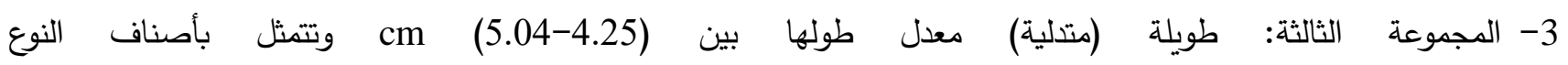
.Dwarf' وهي 'Ming White' macroura أما بالنسبة لعرض الثمرة Width fruit فقد تباينت أيضاً، اذ سجل الصنف 'Rease' للنوع M. alba أعلى معدل له إذ بلغ

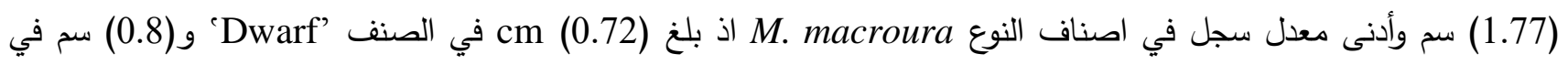
الصنف 'King White' وتدرجت الاصناف الأخرى بين هذه الحدود. وتعد الاختلافات في حجم الثمرة Fruit size من الخصائص التصنيفية المهمة والتي مكنت من تقسيم اصناف أنواع الجنس

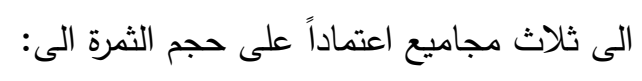

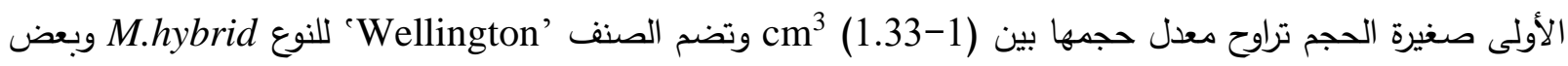
اصناف النوع M.alba وهي 'Preece' و و'Pearl' و 'Pendula`. والثانية متوسطة الحجم والتي معدل حجمها تراوح بين (1.5-

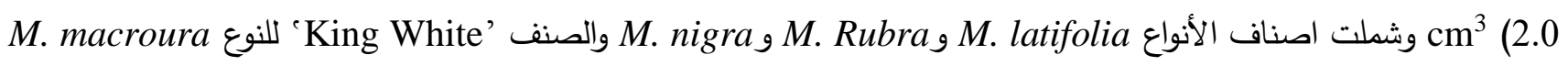
والاصناف 'Beautiful Day و'Border Sweet' و 'Big White' العائدة للنوع M. alba. اما الثالثة كبيرة الحجم والتي تراوح

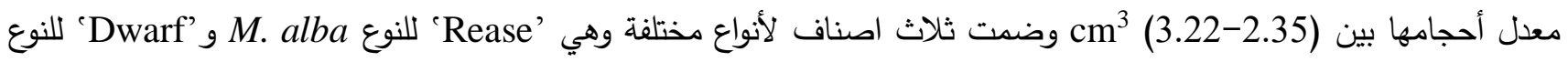

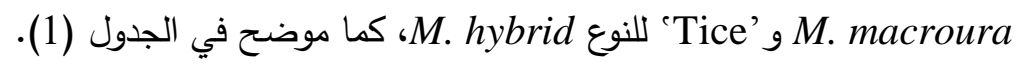


جدول (1) الصفات الكمية والنوعية المميزة لثمار أصناف الأنواع للجنس .Morus Lيد الدراسة

\begin{tabular}{|c|c|c|c|c|c|c|c|c|}
\hline حجم الثمرة cm³ & عرض الثمرة cm & طول الثمرة cm & عدد الثميرات/ثمرة & طول الحامل الثمري & لون الثمرة & شكل الثمرة & الصنف & النوع \\
\hline $\begin{array}{c}* 2.03(2.6-1.4) \\
* * 0.49\end{array}$ & $\begin{array}{c}* 1.4(1.6-1.2) \\
* * 0.10\end{array}$ & $\begin{array}{c}* 2.24(2.8-1.8) \\
* * 0.28\end{array}$ & $\begin{array}{c}* 40.5(52-30) \\
* * 6.77\end{array}$ & $\begin{array}{c}* 0.47(0.8-0.3) \\
* * 0.13\end{array}$ & White & Elliptic & $\begin{array}{l}\text { 'Beautiful } \\
\text { Day' }\end{array}$ & \multirow{7}{*}{ M. alba } \\
\hline $\begin{array}{c}* 1.50(2-1.15) \\
* * 0.23\end{array}$ & $\begin{array}{c}* 1.14(1.24-1) \\
* * 0.07\end{array}$ & $\begin{array}{l}* 2.21(2.45-2) \\
* * 0.12\end{array}$ & $\begin{array}{c}* 62.2(70-53) \\
* * 6.14\end{array}$ & $\begin{array}{c}* 0.59(0.7-0.52) \\
* * 0.07\end{array}$ & White & Elongate Ovoid & 'Big White' & \\
\hline $\begin{array}{c}* 3.22(4.2-2.7) \\
* * 0.58\end{array}$ & $\begin{array}{c}* 1.77(2.2-1.6) \\
* * 0.16\end{array}$ & $\begin{array}{c}* 3.1(4-2.5) \\
* * 0.42\end{array}$ & $\begin{array}{c}* 66.4(78-57) \\
* * 8.88\end{array}$ & $\begin{array}{c}* 0.32(0.5-0.2) \\
* * 0.10\end{array}$ & $\begin{array}{c}\text { White-Light } \\
\text { violet }\end{array}$ & Elliptic & 'Rease' & \\
\hline $\begin{array}{l}* 1.03(1.25-0.7) \\
* * 0.17\end{array}$ & $\begin{array}{c}* 0.92(1.1-0.8) \\
* * 0.07\end{array}$ & $\begin{array}{c}* 1.34(1.7-1) \\
* * 0.18\end{array}$ & $\begin{array}{c}* 34.4(49-26) \\
* * 6.98\end{array}$ & $\begin{array}{c}* 0.49(0.6-0.3) \\
* * 0.087\end{array}$ & $\begin{array}{l}\text { White-Light } \\
\text { violet }\end{array}$ & Ovoid & 'Greece' & \\
\hline $\begin{array}{c}* 1.13(1.2-1) \\
* * 0.06\end{array}$ & $\begin{array}{c}* 0.93(1.2-0.7) \\
* * 0.13\end{array}$ & $\begin{array}{c}* 1.53(1.9-1.2) \\
* * 0.20 \\
\end{array}$ & $\begin{array}{c}* 32.5(43-25) \\
* * 5.82\end{array}$ & $\begin{array}{c}* 0.55(0.8-0.3) \\
* * 0.18\end{array}$ & $\begin{array}{c}\text { White-Light } \\
\text { violet }\end{array}$ & Ovoid & 'Pearl' & \\
\hline $\begin{array}{c}* 1.62(2-1.4) \\
* * 0.25\end{array}$ & $\begin{array}{c}* 1.08(1.2-1) \\
* * 0.11\end{array}$ & $\begin{array}{c}* 1.96(2.3-1.5) \\
* * 0.26\end{array}$ & $\begin{array}{c}* 29.6(37-23) \\
* * 4.58\end{array}$ & $\begin{array}{l}* 0.83(1.2-0.6) \\
* * 0.21\end{array}$ & Violet & Elliptic & 'Border Sweet' & \\
\hline $\begin{array}{c}* 1.1(1.5-0.9) \\
* * 0.21\end{array}$ & $\begin{array}{c}* 1.06(1.3-0.9) \\
* * 0.10\end{array}$ & $\begin{array}{c}* 1.72(2.6-1.3) \\
* * 0.27\end{array}$ & $\begin{array}{c}* 30.3(38-24) \\
* * 4.40\end{array}$ & $\begin{array}{c}* 0.58(1.3-0.4) \\
* * 0.32\end{array}$ & Red-Black & Ovoid & 'Pendula' & \\
\hline $\begin{array}{c}* 1.75(2.1-1.3) \\
* * 0.26\end{array}$ & $\begin{array}{c}* 1.26(1.4-1.15) \\
* * 0.09\end{array}$ & $\begin{array}{c}* 2.35(2.71-2.03) \\
* * 0.21\end{array}$ & $\begin{array}{c}* 44.4(57-30) \\
* * 8.50\end{array}$ & $\begin{array}{c}* 0.99(1.38-0.76) \\
* * 0.17\end{array}$ & Dark violet & Elongate Ovoid & $\begin{array}{l}\text { 'Kokuso } \\
\text { Korean' }\end{array}$ & M.latifolia \\
\hline $\begin{array}{c}* 2.02(2.5-1.7) \\
* * 0.29\end{array}$ & $\begin{array}{c}* 1.22(1.4-1.1) \\
* * 0.09\end{array}$ & $\begin{array}{c}* 2.22(2.9-1.9) \\
* * 0.27\end{array}$ & $\begin{array}{c}* 61.5(76-39) \\
* * 21.51\end{array}$ & $\begin{array}{c}* 0.97(1.5-0.6) \\
* * 0.26\end{array}$ & Red-Black & Elliptic & 'Amarah' & M.rubra \\
\hline $\begin{array}{l}* 2.01(2.4-1.4) \\
* * 0.37\end{array}$ & $\begin{array}{l}* 1.48(1.7-1.3) \\
* * 0.10\end{array}$ & $\begin{array}{l}* 1.83(2.5-1.4) \\
\quad * 0.32\end{array}$ & $\begin{array}{c}* 22.3(30-17) \\
* * 4.06\end{array}$ & sessile & Red-Black & $\begin{array}{c}\text { Semispherical- } \\
\text { Elliptic }\end{array}$ & 'Shami' & M.nigra \\
\hline $\begin{array}{l}* 1.59(2-1) \\
* * 0.34\end{array}$ & $\begin{array}{c}* 0.8(1-0.75) \\
* * 0.06\end{array}$ & $\begin{array}{c}* 4.25(5.4-3.5) \\
* * 0.53\end{array}$ & $\begin{array}{c}* 127.5(169-96) \\
* * 24.68\end{array}$ & $\begin{array}{c}* 1.44(1.7-1) \\
* * 0.19\end{array}$ & $\begin{array}{l}\text { Greenish } \\
\text { yellow }\end{array}$ & Cylindric & 'King White' & \multirow{2}{*}{ M.macroura } \\
\hline $\begin{array}{c}* 2.35(3.5-1.6) \\
* * 0.59\end{array}$ & $\begin{array}{c}* 0.72(0.9-0.6) \\
* * 0.09\end{array}$ & $\begin{array}{c}* 5.04(6.5-3.5) \\
* * 0.84\end{array}$ & $\begin{array}{c}* 66.8(87-46) \\
* * 14.28\end{array}$ & $\begin{array}{c}* 1.5(2-1) \\
* * 0.25\end{array}$ & Red-Black & Cylindric & 'Dwarf' & \\
\hline $\begin{array}{l}* 2.91(3.5-1.9) \\
* * 0.58\end{array}$ & $\begin{array}{c}* 1.69(1.9-1.5) \\
* * 0.12\end{array}$ & $\begin{array}{c}* 2.57(3.1-1.9) \\
* * 0.32\end{array}$ & $\begin{array}{c}* 54.9(74-34) \\
* * 15.2\end{array}$ & $\begin{array}{c}* 1.05(1.5-0.7) \\
* * 0.27\end{array}$ & Red-Black & Elliptic & 'Tice' & \multirow{2}{*}{ M.hybrid } \\
\hline $\begin{array}{c}* 1.33(1.8-0.9) \\
* * 0.28\end{array}$ & $\begin{array}{c}* 1.02(1.2-0.9) \\
* * 0.09\end{array}$ & $\begin{array}{c}* 1.82(2.5-1.2) \\
* * 0.31\end{array}$ & $\begin{array}{c}* 45.3(53-38) \\
* * 5.31\end{array}$ & $\begin{array}{c}* 0.57(0.8-0.3) \\
* * 0.12\end{array}$ & Red-Black & Elongate Ovoid & 'Wellington' & \\
\hline
\end{tabular}




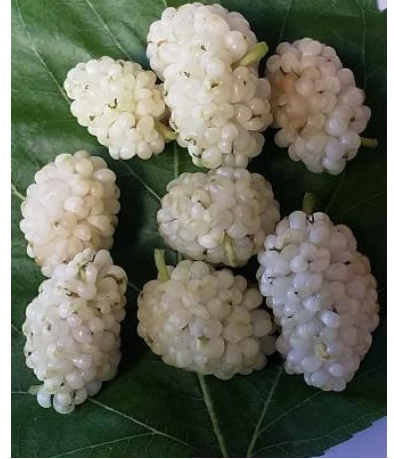

(1)

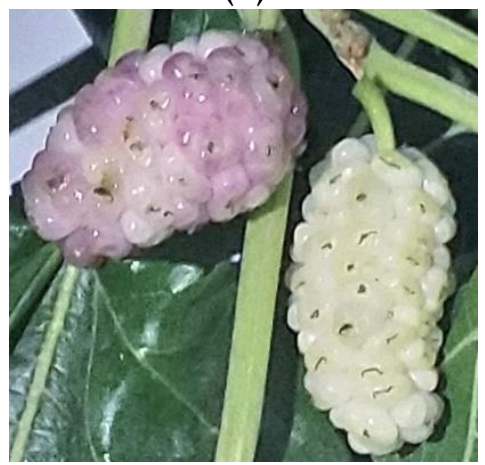

(3)

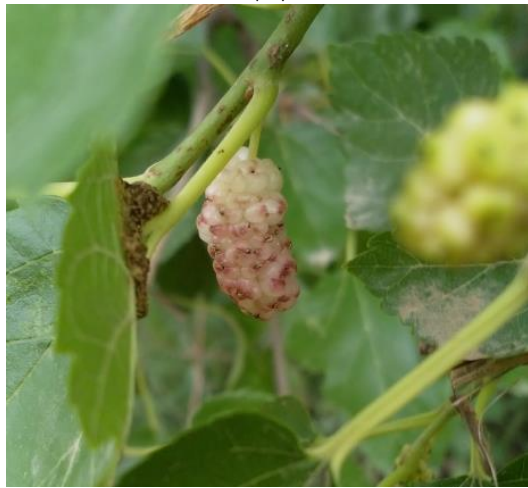

(5)

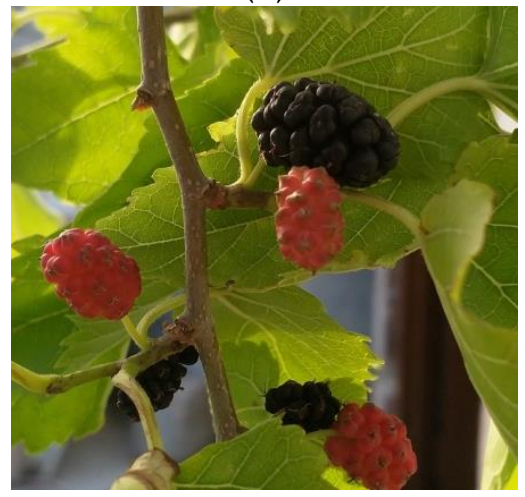

(7)

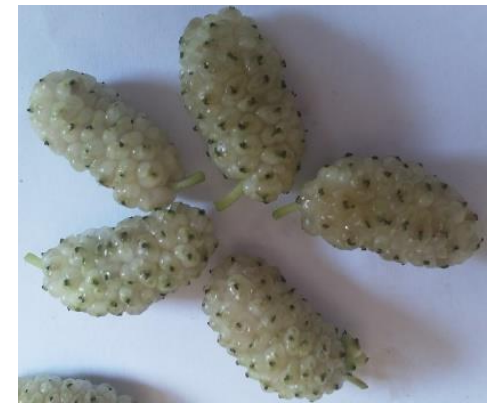

(2)

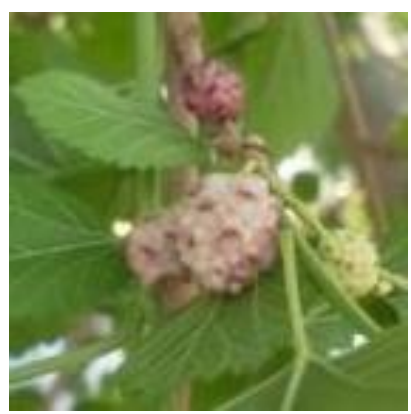

(4)

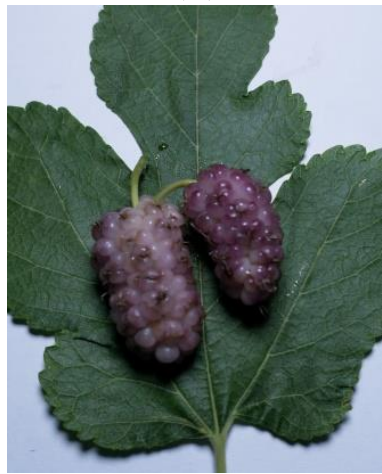

(6)

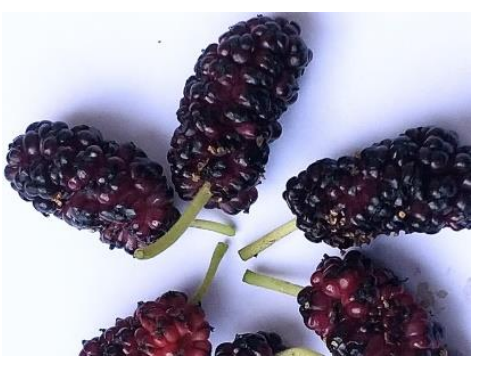

(8)

شكل (1) التغايرات في ثمار أصناف أنواع الجنس . Morus L. قيد الاراسة

1. M. alba 'Beautiful Day'. 2. M. alba 'Big White'. 3. M. alba 'Rease'. 4.M. alba 'Greece'. 5. M. alba 'Pearl'. 6. M. alba 'Border Sweet'. 7. M. alba 'Pendula'. 8.M. latifolia 'KokusoKoreon'. 9.M. rubra 'Amarah'. 10.M. nigra 'Shami'. 11.M. macroura 'King White'. 12.M. macroura 'Dwarf'. 13.M. hybrid 'Tice'. 14. M. hybrid 'Wellington'. 


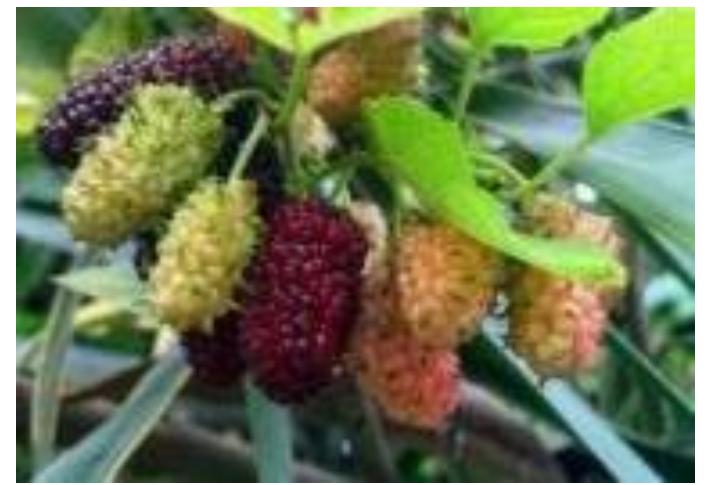

(9)

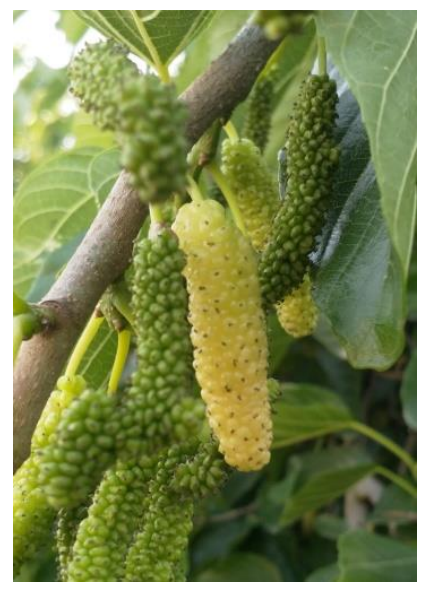

(11)

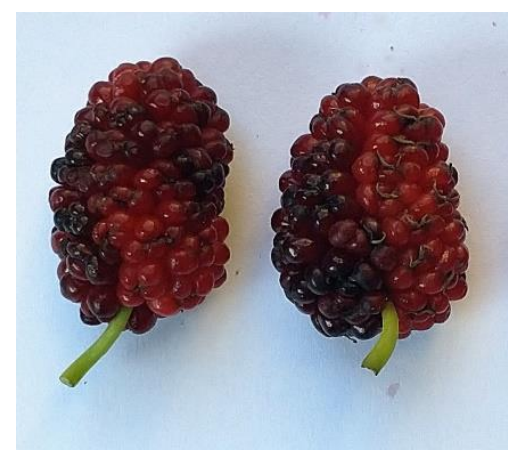

(13)

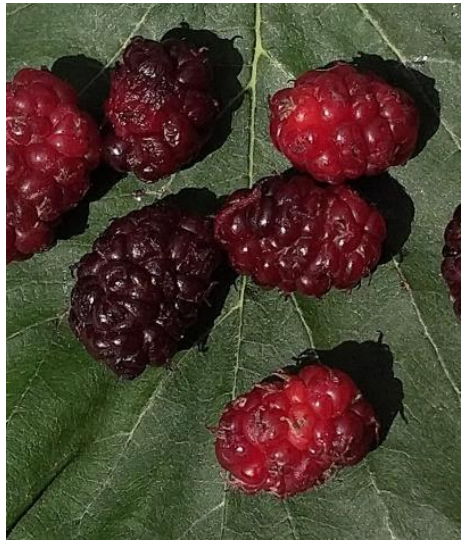

$(10)$

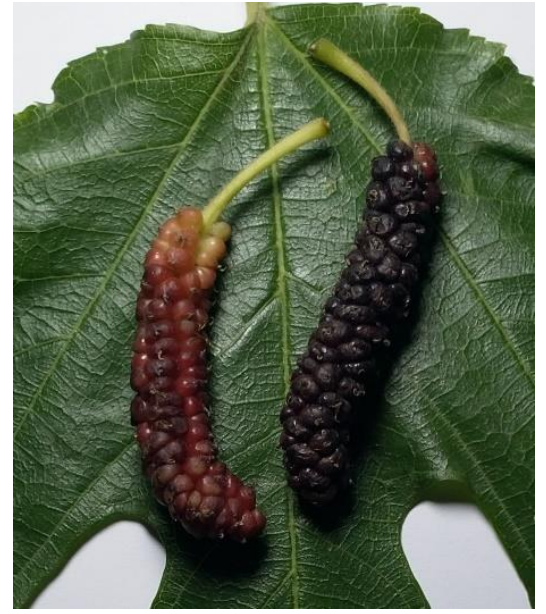

(12)

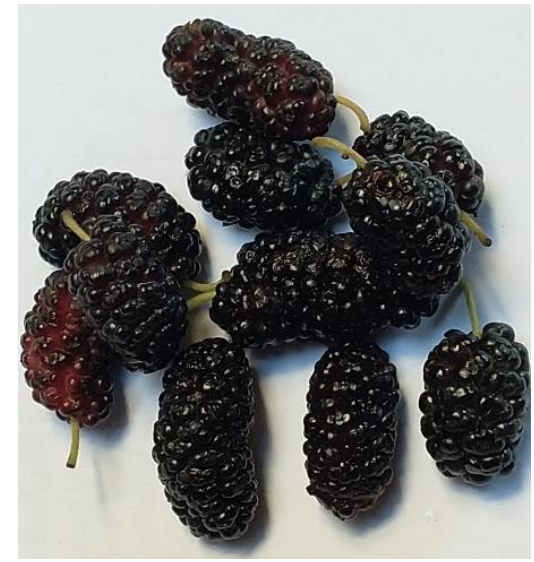

(14)

تابع لثكل (1) التغايرات في ثمار أصناف أنواع الجنس . Morus L قيد الدارة

1. M. alba 'Beautiful Day'. 2. M. alba 'Big White'. 3. M. alba 'Rease'. 4.M. alba 'Greece'. 5. M. alba 'Pearl'. 6. M. alba 'Border Sweet'. 7. M. alba 'Pendula'. 8.M. latifolia 'KokusoKoreon'. 9.M. rubra 'Amarah'. 10.M. nigra 'Shami'. 11.M. macroura 'King White'. 12.M. macroura 'Dwarf'. 13.M. hybrid 'Tice'. 14. M. hybrid 'Wellington'. 
بينت نتائج الدراسة وجود تغايرات واسعة في الخصائص والصفات المظهرية للثمار وحواملها الثمرية في

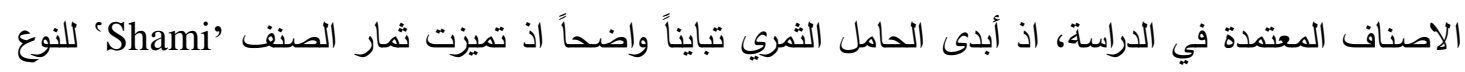

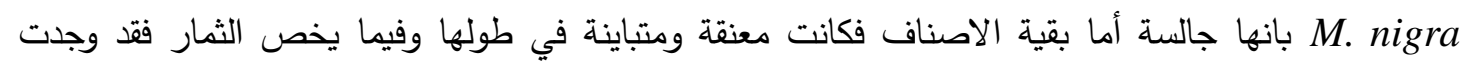

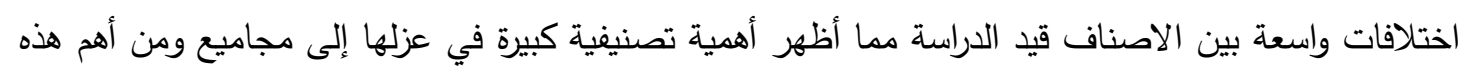

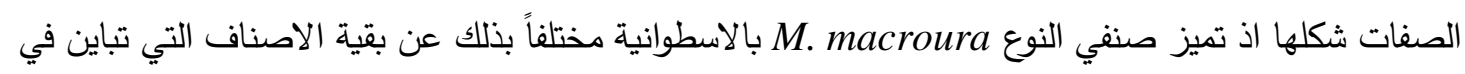

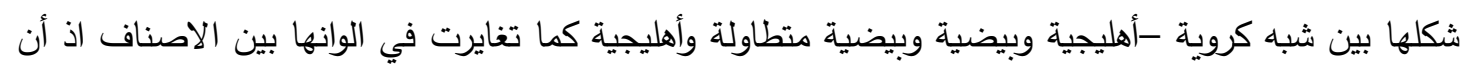

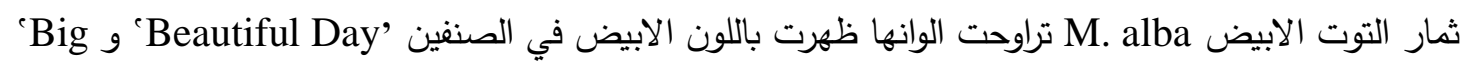

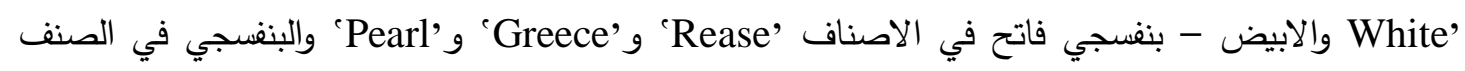

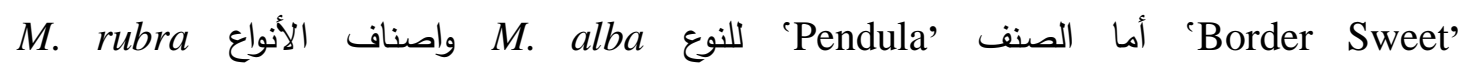

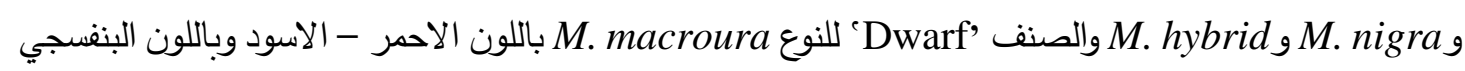
الداكن في الصنف Kokuso Korean للنوع M. latifolia والاصفر المخضر في الصنف CKing White’ الأبيض M. alba سمي باسم لون براعمه، وليس لون ثماره [15].

وتباينت اصناف الأنواع في صفة عدد الثميرات في الثمرة الواحدة اذ سجل في حده الاعلى بمعدل (127.5)

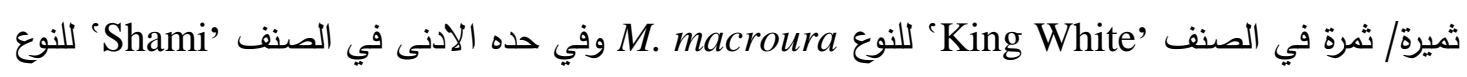
M. nigra إلى مجاميع مما عزز الأهمية التصنيفية للثمار في تثخيص وتمييز اصناف الأنواع التابعة للجنس قيد الدراسة وتتفق هذه النتيجة مع ما اشار اليه الباحثون [12, 14, 16, 17, 18 في وجود اختلافات في الصفات الكمية والنوعية لثمار التوت.

Seed البذرة 2

وضحت الدراسة تغاير في بذور اصناف أنواع الجنس في صفاتها الكمية والنوعية اذ أظهرت تباين واضح في شكلها وابعادها والوانها وزخرفتها السطحية، والتي أمكن تقسيمها حسب شكلها إلى اربع مجاميع كما موضح فئح في الجدول (2) والثكل (2): 1- المجموعة الأولى: ذات شكل بيضي متطاول Elongate ovoid وتضم الصنف 'KokusoKorean' للنوع 'Mellid M.latifolia .'Border Sweet' و 'Pearl' و Big White' و Beautiful Day'

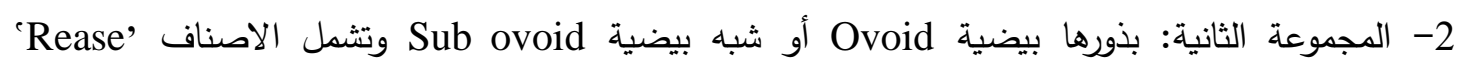

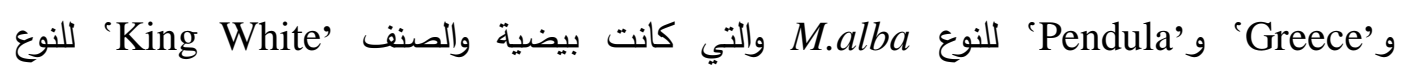
M.macroura

3- المجموعة الثالثة: شبه كروية Sub spherical وشملت الصنف 'Dwarf' للنوع M.macroura. 4- المجموعة الرابعة: بذورها شبه كروية - شبه البيضية Sub spherical-Sub ovoid وضمت اصناف الأنواع M.hybrid والصنف M.nigrabra 
Journal of Education and Science (ISSN 1812-125X), Vol: 29, No: 4, 2020 (177-192)

جدول (2) الصفات الكمية والنوعية المميزة لبذور أصناف أنواع الجنس .Morus L قيد الدراسة

\begin{tabular}{|c|c|c|c|c|c|c|c|c|c|}
\hline |السطحية للبذور & طرض البذرة/ & عدد البذور في الثمرة & سمك البذرة/ملم & عرض البذرة/ ملم & طول البذرة/ ملم & لون البذرة & شكل البذرة & الصنف & النوع \\
\hline $\begin{array}{l}\text { Irregular } \\
\text { reticulate }\end{array}$ & 1.46 & $\begin{array}{c}* 14.6(20-7) \\
* * 7.31\end{array}$ & $\begin{array}{c}* 1.39(1.6-1.2) \\
* * 0.11\end{array}$ & $\begin{array}{c}* 1.95(2.1-1.75) \\
* * 0.11\end{array}$ & $\begin{array}{c}* 2.85(3-2.6) \\
* * 0.15\end{array}$ & Light brown & $\begin{array}{c}\text { Elongate } \\
\text { ovoid }\end{array}$ & 'Beautiful Day' & \multirow{7}{*}{ M. alba } \\
\hline $\begin{array}{l}\text { Irregular } \\
\text { reticulate }\end{array}$ & 1.62 & $\begin{array}{c}* 59.1(70-47) \\
* * 7.28\end{array}$ & $\begin{array}{c}* 1.10(1.3-0.9) \\
* * 0.13\end{array}$ & $\begin{array}{c}* 1.56(1.7-1.4) \\
* * 0.12\end{array}$ & $\begin{array}{c}* 2.25(2.8-2.2) \\
* * 0.18\end{array}$ & Light brown & $\begin{array}{c}\text { Elongate } \\
\text { ovoid }\end{array}$ & 'Big White' & \\
\hline $\begin{array}{l}\text { Irregular } \\
\text { reticulate }\end{array}$ & 1.48 & $\begin{array}{c}* 53.7(73-35) \\
* * 12.83 \\
\end{array}$ & $\begin{array}{c}* 1.31(1.7-1.1) \\
* * 0.15\end{array}$ & $\begin{array}{c}* 1.69(1.85-1.5) \\
* * 0.12 \\
\end{array}$ & $\begin{array}{l}* 2.5(2.75-2.25) \\
* * 0.18 \\
\end{array}$ & $\begin{array}{c}\text { Yellowish } \\
\text { brown }\end{array}$ & Ovoid & 'Rease' & \\
\hline $\begin{array}{l}\text { Regular } \\
\text { reticulate }\end{array}$ & 1.61 & $\begin{array}{c}* 29.5(39-20) \\
* * 5.70\end{array}$ & $\begin{array}{c}* 1.10(1.3-1) \\
* * 0.11\end{array}$ & $\begin{array}{c}* 1.42(1.5-1.2) \\
* * 0.10\end{array}$ & $\begin{array}{c}* 2.28(2.35-2) \\
* * 0.11\end{array}$ & Light brown & Ovoid & 'Greece' & \\
\hline $\begin{array}{c}\text { Regular } \\
\text { reticulate }\end{array}$ & 1.42 & $\begin{array}{c}* 17.4(40-7) \\
* * 9.11\end{array}$ & $\begin{array}{c}* 1.11(1.4-0.8) \\
* * 0.16\end{array}$ & $\begin{array}{c}* 1.26(1.5-1) \\
* * 0.15\end{array}$ & $\begin{array}{c}* 1.79(2-1.4) \\
* * 0.17\end{array}$ & Light brown & $\begin{array}{c}\text { Elongate } \\
\text { Ovoid }\end{array}$ & 'Pearl' & \\
\hline $\begin{array}{c}\text { Rugose } \\
\text { reticulate }\end{array}$ & 1.36 & $\begin{array}{c}* 16(28-8) \\
* * 7.39\end{array}$ & $\begin{array}{c}* 1.51(1.75-1.4) \\
* * 0.11\end{array}$ & $\begin{array}{c}* 1.64(1.8-1.4) \\
* * 0.14\end{array}$ & $\begin{array}{c}* 2.23(2.7-1.7) \\
* * 0.31\end{array}$ & Dark brown & $\begin{array}{c}\text { Elongate } \\
\text { ovoid }\end{array}$ & 'Border Sweet' & \\
\hline $\begin{array}{c}\text { Regular } \\
\text { reticulate }\end{array}$ & 1.54 & $\begin{array}{l}* 10(19-4) \\
* * 5.27\end{array}$ & $\begin{array}{c}* 1.25(1.4-1.1) \\
* * 0.09\end{array}$ & $\begin{array}{c}* 1.56(2-1.4) \\
* * 0.18\end{array}$ & $\begin{array}{c}* 2.41(2.8-2.1) \\
* * 0.20\end{array}$ & Light brown & Ovoid & 'Pendula' & \\
\hline $\begin{array}{l}\text { Micro } \\
\text { reticulate }\end{array}$ & 1.53 & $\begin{array}{c}* 42.4(52-30) \\
* * 6.77\end{array}$ & $\begin{array}{c}* 1.50(1.6-1.3) \\
* * 0.10\end{array}$ & $\begin{array}{c}* 1.87(2-1.65) \\
* * 0.14\end{array}$ & $\begin{array}{c}* 2.86(3-2.7) \\
* * 0.11\end{array}$ & Light brown & $\begin{array}{c}\text { Elongate } \\
\text { ovoid }\end{array}$ & 'Kokuso Korean' & M.latifolia \\
\hline $\begin{array}{l}\text { Papillate } \\
\text { ruminate }\end{array}$ & 1.34 & $\begin{array}{c}* 3.8(13-0) \\
* * 3.94\end{array}$ & $\begin{array}{c}* 1.12(1.4-1) \\
* * 0.16\end{array}$ & $\begin{array}{c}* 1.73(1.9-1.5) \\
* * 0.14\end{array}$ & $\begin{array}{c}* 2.31(2.4-2.2) \\
* * 0.07\end{array}$ & $\begin{array}{l}\text { Yellowish } \\
\text { brown }\end{array}$ & $\begin{array}{c}\text { Sub spherical- } \\
\text { Sub ovoid }\end{array}$ & 'Amarah' & M.rubra \\
\hline Muricate & 1.31 & $\begin{array}{l}* 3.9(7-1) \\
* * 1.91\end{array}$ & $\begin{array}{c}* 1.16(1.3-1) \\
* * 0.09\end{array}$ & $\begin{array}{c}* 2.47(2.7-2.25) \\
* * 0.19\end{array}$ & $\begin{array}{c}* 3.23(3.7-2.85) \\
* * 0.26\end{array}$ & $\begin{array}{l}\text { Blackish } \\
\text { brown }\end{array}$ & $\begin{array}{c}\text { Sub spherical- } \\
\text { Sub ovoid }\end{array}$ & 'Shami' & M.nigra \\
\hline $\begin{array}{l}\text { Foveate } \\
\text { ruminate }\end{array}$ & 1.44 & $\begin{array}{l}* 1.5(5-0) \\
* * 2.07\end{array}$ & $\begin{array}{c}* 1.54(1.8-1.35) \\
* * 0.21\end{array}$ & $\begin{array}{c}* 1.1(1.3-0.95) \\
* * 0.11\end{array}$ & $\begin{array}{c}* 1.58(1.7-1.3) \\
* * 0.13\end{array}$ & Light yellow & Sub ovoid & 'King White' & \multirow{2}{*}{ M.macroura } \\
\hline $\begin{array}{l}\text { Foveate } \\
\text { undulate }\end{array}$ & 1.19 & $\begin{array}{l}* 3.6(13-0) \\
* * 5.08\end{array}$ & $\begin{array}{c}* 0.85(0.9-0.75) \\
* * 0.05\end{array}$ & $\begin{array}{c}* 1.35(1.5-1.2) \\
* * 0.10\end{array}$ & $\begin{array}{c}* 1.61(1.7-1.45) \\
* * 0.08\end{array}$ & Light yellow & $\begin{array}{c}\text { Sub spherical- } \\
\text { Sub ovoid }\end{array}$ & 'Dwarf' & \\
\hline Polygonal & 1.30 & $\begin{array}{l}* 4.2(12-1) \\
\quad * * 3.35\end{array}$ & $\begin{array}{c}* 0.97(1.1-0.85) \\
* * 0.07\end{array}$ & $\begin{array}{c}* 2.4(2.75-2.1) \\
* * 0.22\end{array}$ & $\begin{array}{c}* 3.13(3.5-2.6) \\
* * 0.26\end{array}$ & $\begin{array}{l}\text { Blackish } \\
\text { brown }\end{array}$ & $\begin{array}{c}\text { Sub spherical- } \\
\text { Sub ovoid }\end{array}$ & 'Tice' & \multirow{2}{*}{ M.hybrid } \\
\hline $\begin{array}{l}\text { Regular } \\
\text { reticulate }\end{array}$ & 1.57 & $\begin{array}{c}* 39.2(47-28) \\
* * 6.37\end{array}$ & $\begin{array}{c}* 1.48(1.85-1.2) \\
* * 0.21\end{array}$ & $\begin{array}{c}* 1.47(1.65-1.3) \\
* * 0.12 \\
\end{array}$ & $\begin{array}{c}* 2.31(2.55-2.2) \\
* * 0.11\end{array}$ & Dark brown & $\begin{array}{c}\text { Elongate } \\
\text { ovoid }\end{array}$ & 'Wellington' & \\
\hline
\end{tabular}




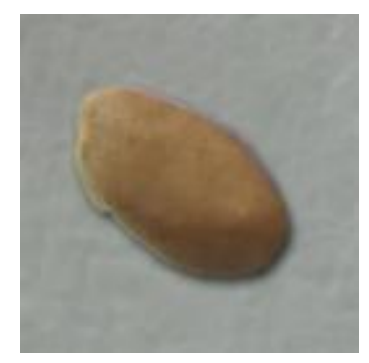

Elongate ovoid $(1,2,5,6,8,14)$

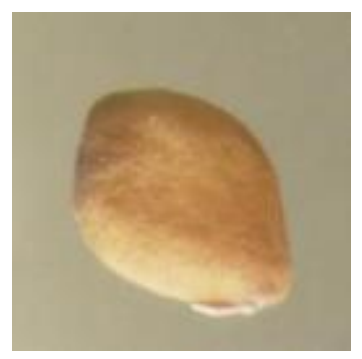

Ovoid - sub Ovoid $(3,4,7,11)$

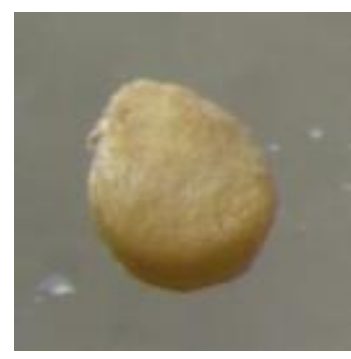

Subspherical (12)

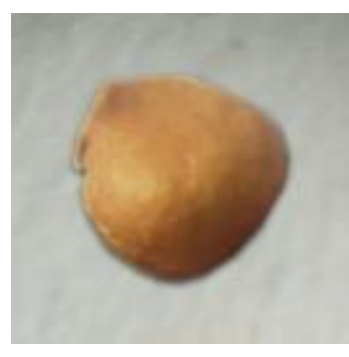

Subspherical-sub ovoid $(9,10,13)$

الثكل (2) التغايرات في شكل بذور أصناف أنواع الجنس .Morus L) قيد الاراسة (قوة التكبير MX)

1. M. alba 'Beautiful Day'. 2. M. alba 'Big White'. 3. M. alba 'Rease'. 4.M. alba 'Greece'. 5. M. alba 'Pearl'. 6. M. alba 'Border Sweet'. 7. M. alba 'Pendula'. 8.M. latifolia 'KokusoKoreon'. 9.M. rubra 'Amarah'. 10.M. nigra 'Shami'. 11.M. macroura 'King White'. 12.M. macroura 'Dwarf'. 13.M. hybrid 'Tice'. 14. M. hybrid 'Wellington'.

ومن حيث لون البذور Colour seeds فكان اللون اصفر فاتح Light yellow في صنفي النوع M.macroura

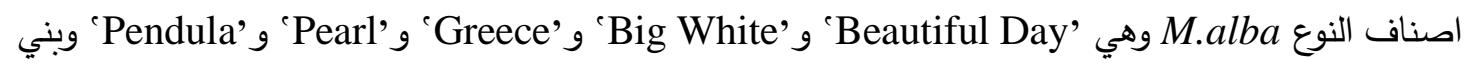

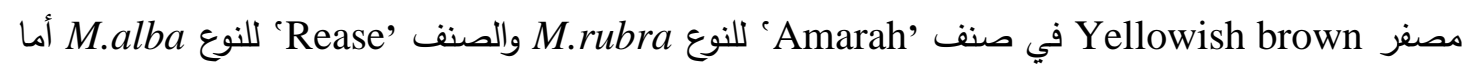

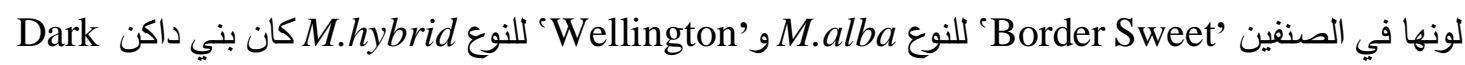

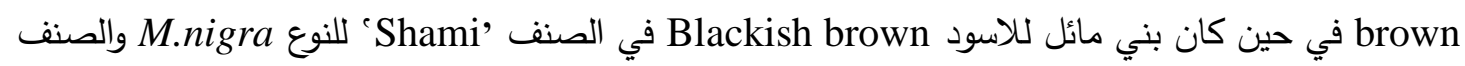
.M.hybrid للنوع 'Tice'

أما من حيث ابعادها واعدادها في الثرة فاختلفت باختلاف الأصناف، اذ وجد أعلى معدل لابعادها (3.23

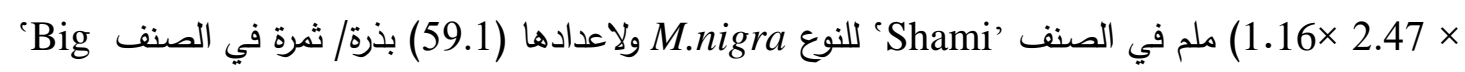
للنوع M.alba White'

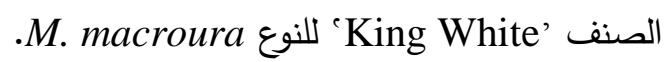

أما بالنسبة لكساءها السطحي فتباين اصناف الانواع في الزخرفة السطحية للبذور

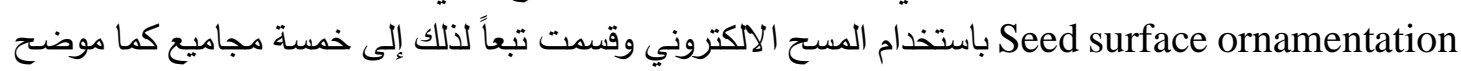
في الجدول (2) والثكل (3) وهي:

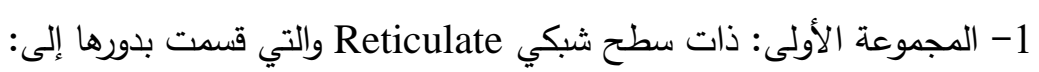
شبكي منتظم Regular reticulate وتضم الاصناف 'Areece' و'Pearl' و 'Pendula' للنوع 'We'

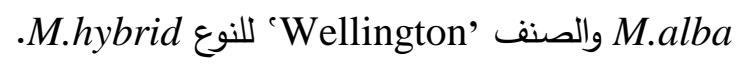

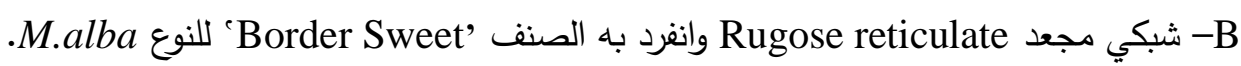
M.latifolia في الصنف 'Mokuso Korean' للنوع دقيق Micro reticulate -C M.alba شتبكي غير منتظم Irregular reticulate وتشمل بقية الاصناف للنوع -D

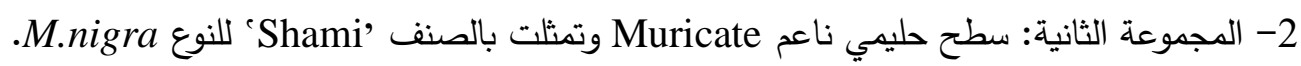


3- المجموعة الثالثة: متموج منقر Foreate undulate وظهر به الصنف 'Dwarf' للنوع M.macroura. 4- المجموعة الرابعة: مضلع الثكل Polygonal وتضم الصنف 'Tice' للنوع M.hybrid.

$$
\text { 5- المجموعة الخامسة: منسحب Ruminate، والتي انقست إلى مسع: }
$$

M.rubra Amarah' في الصنف Papillate ruminate منسحب حليمي -a

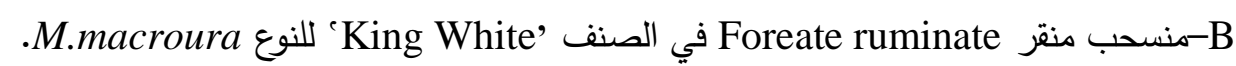

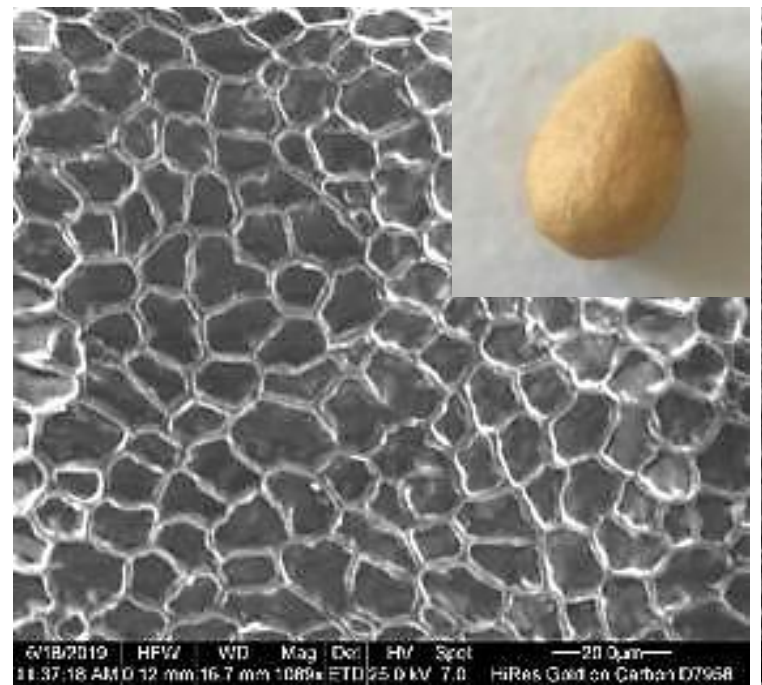

Regular reticulate

$(4,5,7,14)$

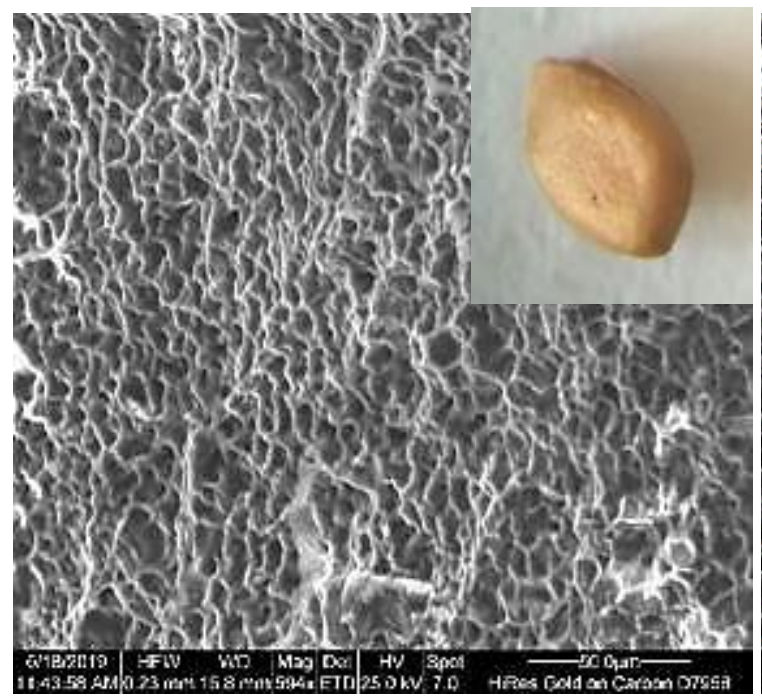

Micro reticulate

(8)

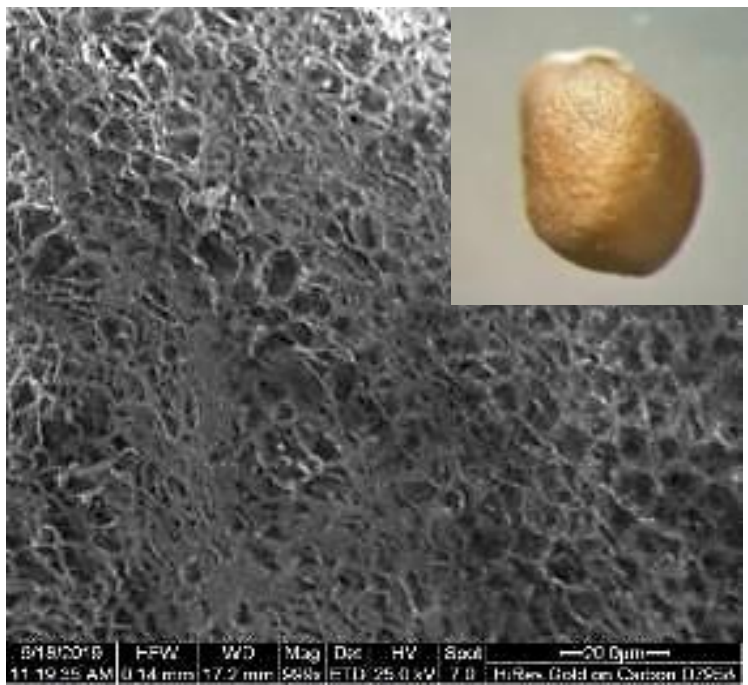

Rugose reticulate

(6)

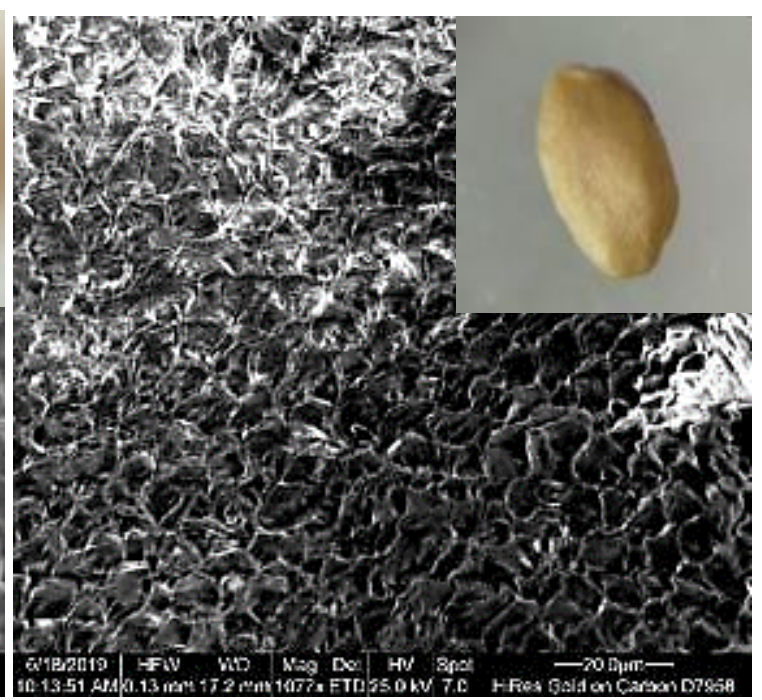

Irregular reticulate

$(1,2,3)$

شكل (3) التغاير في الزخرفة السطحية لبذور أصناف أنواع الجنس .Morus L قيد الدراسة باستخدام المجهر التشريحي (15X) والمجهر الالكتروني الماسح (SEM)

1. M. alba 'Beautiful Day'. 2. M. alba 'Big White'. 3. M. alba 'Rease'. 4.M. alba 'Greece'. 5. M. alba 'Pearl'. 6. M. alba 'Border Sweet'. 7. M. alba 'Pendula'. 8.M. latifolia 'KokusoKoreon'. 9.M. rubra 'Amarah'. 10.M. nigra 'Shami'. 11.M. macroura 'King White'. 12.M. macroura 'Dwarf'. 13.M. hybrid 'Tice'. 14. M. hybrid 'Wellington'. 


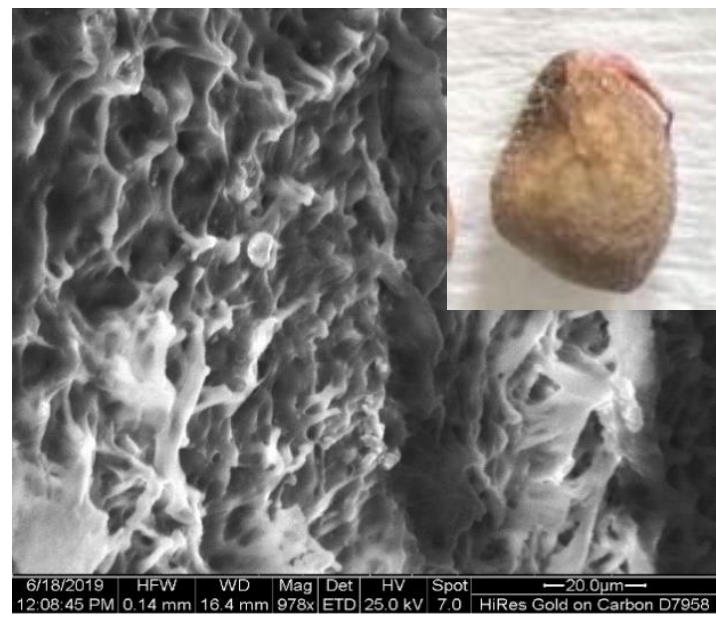

Muricate

(10)

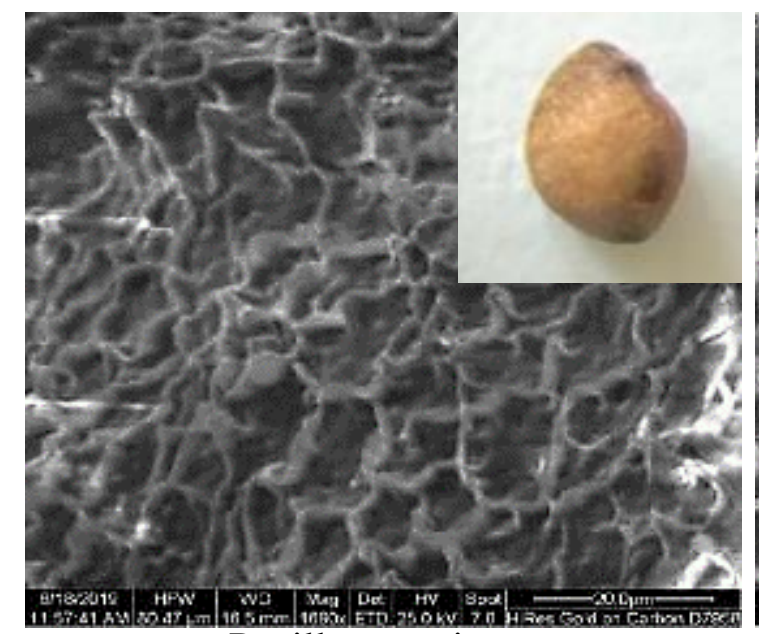

Papillate ruminate

(9)

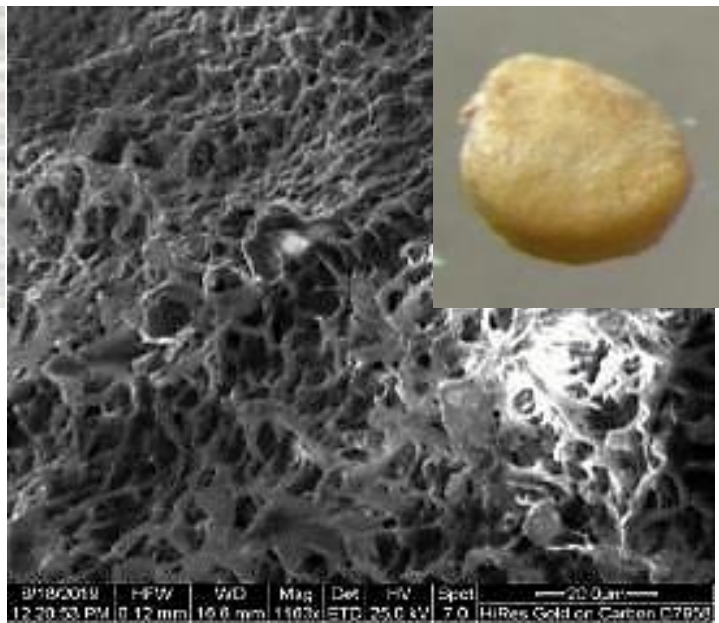

Foreate Undulte

(12)

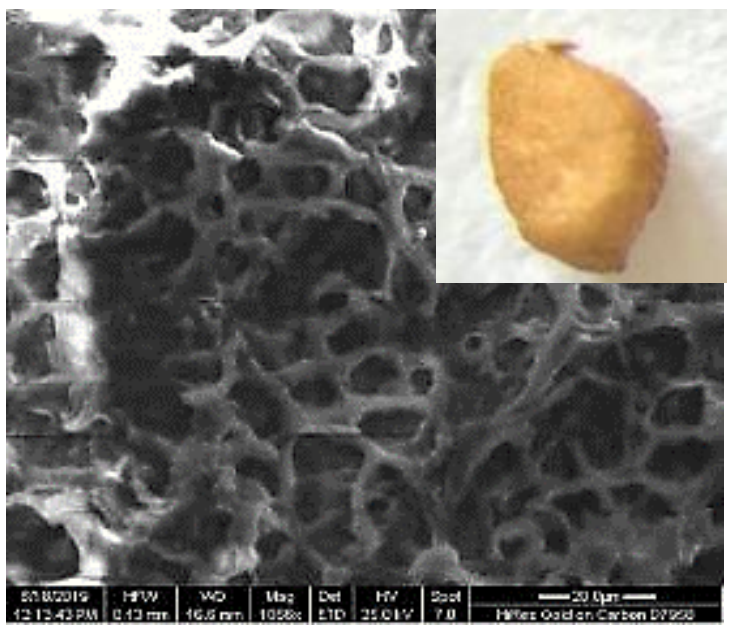

Foreate ruminate

(11)

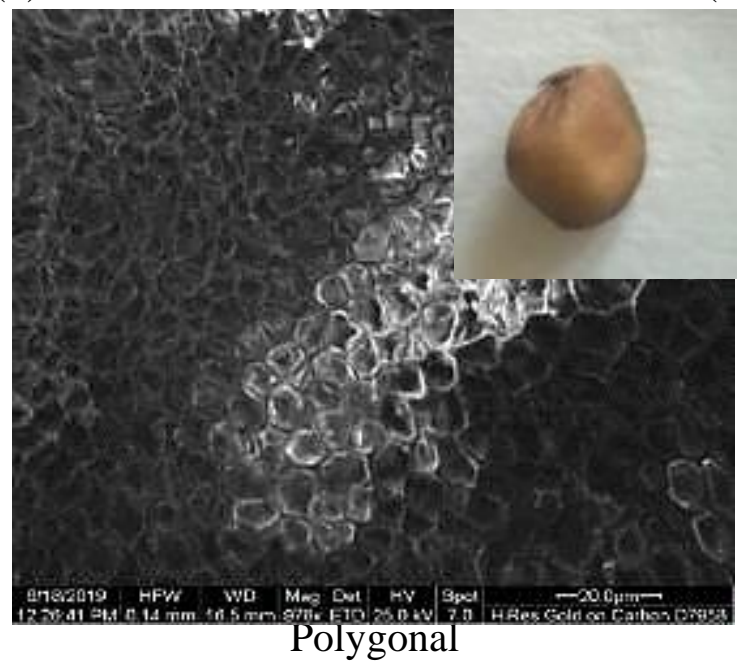

(13)

تابع شكل (3) التغاير في الزخرفة السطحية لبذور أصناف أنواع الجنس .Morus L قيد الدراسة باستخدام

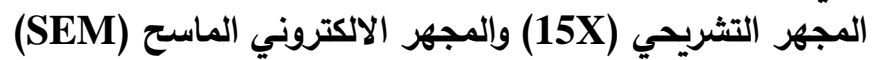

1. M. alba 'Beautiful Day'. 2. M. alba 'Big White'. 3. M. alba' 'Rease'. 4.M. alba 'Greece'. 5. M. alba 'Pearl'. 6. M. alba 'Border Sweet'. 7. M. alba 'Pendula'. 8.M. latifolia 'KokusoKoreon'. 9.M. rubra 'Amarah'. 10.M. nigra 'Shami'. 11.M. macroura 'King White'. 12.M. macroura 'Dwarf'. 13.M. hybrid 'Tice'. 14. M. hybrid 'Wellington'. 
أن للبذور قيمة ومنزلة تصنيفية نظراً للاختلافات التي أبدتها في خصائصها المظهرية فمن حيث اشكالها فقد كانت بيضية منطاولة وبيضية أو شبه بيضية عدا اصناف الأنواع M. nigra, M. rubra والصنف 'Tice' للنوع Mybrid فكانت بذورها شبه كروية - شبه بيضية فيما انفرد صنف 'Dwarf' للنوع M. macroura

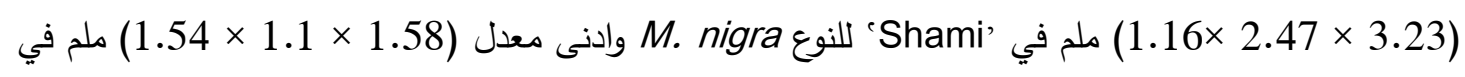
الصنف 'King White' للنوع M. macroura ونظراً لأن طول البذرة كان أقل من 5 ملم، فأن هذا يضع البذرة في فئة (A) لحجم البذور التي اقترحها [19] وتتفق هذه النتائج دع اشار اليه [21،20]. أما بالنسبة لعدد البذور في الثمرة فقد تغايرت الاصناف بثكل ملحوظ اذ وجد أن أعلى عددها في ثرة

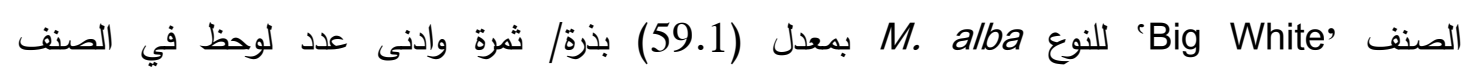

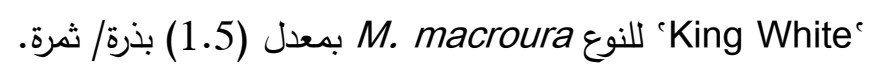
وفيما يتعلق بالكساء السطحي للبذور اظهر الفحص بالمجهر الاككتروني اختلافات واسعة بين الاصناف امكن من عزلها الى عدة مجاميع حسب نوع الزخرفة السطحية (تثكل الثمع) للبذور مما عزز وبشكل واضح الأهمية التصنيفية للبذور في امكان اعتماد صفاتها في تثخيص وتصنيف اصناف الأنواع للجنس قيد الدراسة

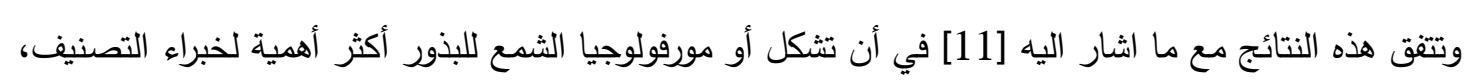
ويمكن أن يكون وسيلة للتمييز بين الاصناف كما تتقق مع [22] في أن التباينات في صفات البذور تتضمن الاختلافات في حجم البذور واشكالها اذ يعتبر شكل البذرة صفة مهمة في تثخيص النبات وتصنيفه. الاستنتاجات 1. يمكن الاعتماد على الصفات المظهرية الكمية والنوعية للثمار والبذور في تثخيص وتمييز اصناف انواع الجنس .Morus L والتي اظهرت ان الصنف Shamr ' للنوع M.nigra اكثر الاصناف اختلافاً في صفاته عن اصناف الانواع الاخرى قيد الدراسة.

2. ان لون الثمار لا يحدد نوع التوت فأصناف التوت الابيض M. alba اعطت ثمار ذات لون ابيض او ابيض - بنفسجي فاتح أو بنفسي او احمر - اسود والتوت الأسود M. nigra اعطى ثمار ذات لون احمر - اسود. 3. ان الزخرفة السطحية للبذور باستخدام المسح الالكتروني من الصفات المهمة التي يستتد اليها في الفصل بين اصناف انواع الجنس قيد الدراسة. 
[1] Kafkas, S., Ozgen, M., Dogan, Y., Ozcan, B., Ercisli, S., Serce, S. J. amer. Soc. Hort. Sci., 133(4):593-597.(2008).

[2] Wani, S. A. Ph. D. Thesis. Sher-e-Kashmir Univ. of Agric, Sci. Tech. Kashmir.(2012).

[3] Radford, A. E., Dikison, W. C., Massey, J.R., Bell, C.R. Harper and Row, New York. p.891.(1974).

[4] Judd, W.S. Campbell, C.S., Kellogg, F.A., Stevens, P.F. Inc. Publishers Sunderland Massachusetts, USA. 1:464P.(1999).

[5] Yilmaz, K.U., Zengin, Y., Ercisli, S., Demirtas, M.N., Kan ,T., Nazli, A.R. J. Anim. Plant sci., 22(1):211-214.(2012).

[6] Lo, B.R., Mirabella, F. Agriclture., 8(10)157: 1-9.(2018).

[7] Galla, S. J. Viers, B. L., Gradie, P. E.; Saar, D. E. Phytologia, 91(1): 105116.(2009).

[8] Teba, Kh. M. and Diop, M. A. Agricultural Scientific Research, Syria, P. 136.(2015). (In Arabic).

[9] Al-Muswi, A. H. University of Mosul, Iraq P. 379. (1987). (In Arabic).

[10] Reino-Molino, J. J., Montejo-valdes, L.A. Sanchez-Rendon, J.A., Martin, G. J. Pastosforrajes, 40(4):259-263.(2017).

[11] Biasiolo, M., Dacanal, M.T., Tornadore, N. Eco. Bot., 58(4):636-646.(2004).

[12] Al-Jane, F. and Sdiri, N. J. New Sci. Agri. Biotechnol., 35 (1): 1940-1947.(2016).

[13] Makhoul; G., Hafiz, M., Husam, B. Teshreem University Journal for Research and scientific studies- Biological sciences series Vol. 39, No, 1: 167-183. (2017). (In Arabic).

[14] Krishna, H., Singh, D., Singh, R.S.,Kumar, L., Sharma, B.D., Saroj, P.L. J. Saudi Soci. Agricult. Sci, 19(2):136-145. (2018).

[15] Facciola S., Comucopia. Kompany Publ., Vista, California, USA, P.ix. 676. (1990).

[16] Jalikop, S. H., Shivashankora. K. S, Kumar. R. ActaHortic. 890:267- 272.(2011). 
[17] Peris, N. W., Gacheri, K. M., Theophillus, M.M., Lucas, N. Agri. Res., 3(1): 1015.(2014).

[18] Balik, A., Gerer, M. K., Aslantas, R. Turk J. Agric.For., 43:28-35.(2019).

[19] Hladik, A. and Miquel, S. MAB, Parthennon publishing Group, P. 261-276.(1990).

[20] Fnafc, A. Flora of north America north to Mexico, 3:390-392.(1997).

[21] Valdes, L. L., Borroto, O. G., Perez, G. F. Food and agriculture organization of the united nations instituto de ciencia animal, Cuba, p.280. (2017).

[22] Gervantes, E., Martin, J.J., Saadaoui, E. Hindawi Publishing Corporation Scientifica, ID: 5691825, 10 Pages.(2016). 